\title{
Maternal aripiprazole exposure interacts with 7-dehydrocholesterol reductase mutations and alters embryonic neurodevelopment
}

\author{
Thiago C. Genaro-Mattos ${ }^{1} \cdot$ Luke B. Allen $^{2} \cdot$ Allison Anderson $^{1} \cdot$ Keri A. Tallman ${ }^{3} \cdot$ Ned A. Porter $^{3} \cdot$ Zeljka Korade $^{2}$. \\ Károly Mirnics $\mathbb{1}^{1}$
}

Received: 25 September 2018 / Revised: 10 December 2018 / Accepted: 23 January 2019 / Published online: 11 February 2019

(c) The Author(s) 2019. This article is published with open access

\begin{abstract}
Mutations in both copies in the gene encoding 7-dehydrocholesterol reductase (DHCR7) cause Smith-Lemli-Opitz Syndrome (SLOS), which is characterized by a toxic elevation in 7-dehydrocholesterol (7-DHC). Aripiprazole (ARI) exposure, independent of genetic mutations, also leads to elevation of 7-DHC. We investigated the combined effect of a single-copy $\mathrm{Dhcr} \mathrm{7}^{+/-}$mutation and maternal ARI exposure on the developing offspring brain. We generated a time-pregnant mouse model where WT and $D h c r 7^{+/-}$embryos were maternally exposed to ARI or vehicle (VEH) from E12 to E19 (5 mg/ $\mathrm{kg}$ ). Levels of cholesterol, its precursors, ARI and its metabolites were measured at P0. We found that ARI and its metabolites were transported across the placenta and reached the brain of offspring. Maternal ARI exposure led to decreased viability of embryos and increased 7-DHC levels, regardless of maternal or offspring $D h c r 7$ genotype. In addition, $D h c r 7^{+/-}$ pups were more vulnerable to maternal ARI exposure than their WT littermates, and maternal $D h c r 7^{+/-}$genotype also exacerbated offspring response to ARI treatment. Finally, both 7-DHC levels and 7-DHC/cholesterol ratio is the highest in $D h c r 7^{+/-}$pups from $D h c r 7^{+/-}$mothers exposed to ARI, underscoring a potentially dangerous interaction between maternal genotypexembryonic genotypextreatment. Our findings have important clinical implications. SLOS patients should avoid drugs that increase 7-DHC levels such as ARI, trazodone and haloperidol. In addition, treatment with 7-DHC elevating substances might be potentially unsafe for the $1-1.5 \%$ of population with single-allele disruptions of the DHCR7 gene. Finally, prenatal and parental genetic testing for DHCR7 should be considered before prescribing sterol-interfering medications during pregnancy.
\end{abstract}

\section{Introduction}

Proper cholesterol metabolism is essential for normal brain function. Cholesterol serves not only as a membrane component but also as a precursor for bile acids, hormones, and

Supplementary information The online version of this article (https:// doi.org/10.1038/s41380-019-0368-6) contains supplementary material, which is available to authorized users.

Károly Mirnics

karoly.mirnics@unmc.edu

1 Munroe-Meyer Institute, University of Nebraska Medical Center, Omaha, NE, USA

2 Department of Pediatrics, University of Nebraska Medical Center, Omaha, NE, USA

3 Department of Chemistry, Vanderbilt University, Nashville, TN, USA other biologically relevant metabolites [1,2]. Owing to the impermeability of the blood-brain barrier to cholesterol, it needs to be synthesized in situ [3] in a sequence of complex enzymatic reactions, which uses acetyl-CoA units to make cholesterol [4]. Altered cholesterol metabolism is linked to a variety of diseases, including genetic neurodevelopmental disorders [5-8]. One of them is Smith-Lemli-Opitz Syndrome (SLOS), an inherited neurodevelopmental disorder characterized by multiple congenital malformations and defects, photosensitivity, impaired cognitive function, and behaviors characteristic of autism spectrum disorders [7, 9].

SLOS is caused by mutations in both copies in the gene encoding the last enzyme in the cholesterol biosynthesis pathway-7-dehydrocholesterol reductase (DHCR7) (Scheme 1, supplemental material) [7, 10-12]. The most significant biochemical change seen in SLOS patients is the dramatic elevation in 7-dehydrocholesterol (7-DHC) [7, 13, 14]. This compound is highly oxidizable and toxic to cells, affecting neuronal viability, proliferation, and 
differentiation, thus contributing to the pathophysiology of the disorder [15-19]. It is believed that heterozygous DHCR7 mutation carriers (parents of SLOS children) have $1-1.5 \%$ frequency in the human population, and they are considered healthy $[20,21]$. However, it has been recently reported that dermal fibroblasts from heterozygous carriers show elevated 7-DHC levels when compared to cells from individuals carrying the $\mathrm{DHCR} 7^{+/+}$genotype [22].

In addition to genetic alterations in the cholesterol biosynthesis, many chemicals are able to disrupt different steps of the cholesterol biosynthesis pathway [23-27]. A screen of the NIH small molecule collection revealed that in cultured cells $\sim 5 \%$ of the compounds elevate 7 -DHC by inhibiting DHCR7 [24]. Interestingly, antipsychotics are among the most potent 7-DHC elevators, including aripiprazole (ARI), an atypical antipsychotic that was the medication with the highest gross sales in 2013 and 2014 in the US (http://www.drugs.com/stats/abilify). ARI increases 7-DHC levels both in vitro and in vivo [22, 23, 28] and inhibits the de novo cholesterol synthesis [29]. In addition, a recent report used primary human fibroblasts to show that the combination of the two 7-DHC-elevating mechanisms (genetic and environmental) results in much higher 7-DHC levels [22], suggesting an unwanted and potentially dangerous synergism between these mechanisms. This study revealed that an exposure to the same concentrations of ARI results in higher levels of 7-DHC in cells with a $D H C R 7^{+/-}$genotype when compared to $D H C R 7^{+/+}$cells. As a conclusion, it was suggested that cells with $D H C R 7^{+/-}$genotype are more vulnerable to ARI's unwanted side effects on cholesterol biosynthesis.

The vulnerability of individuals with a $\mathrm{DHCR} 7^{+/-}$genotype to side effects of ARI has potential clinical implications, as (1) cholesterol has to be synthesized de novo during embryonic development [2, 4]; (2) ARI is often prescribed to pregnant women [30-35]; 3) ARI is transported across the placenta and crosses the blood-brain barrier [36, 37]; (4) ARI disrupts the cholesterol biosynthesis and elevates 7DHC levels in the toxic range [22-24, 28]; (5) $1-1.5 \%$ of the population carries a $\mathrm{DHCR} 7^{+/-}$mutation and might be more vulnerable to this medication. Based on these facts, we hypothesized that maternal ARI exposure will inhibit DHCR7 and increase 7-DHC in the developing brain of offspring, posing a serious risk to embryonic development. Furthermore, we proposed that embryos that carry a singlecopy $\mathrm{DHCR} 7^{+/-}$mutation would show enhanced sensitivity to intrauterine ARI exposure. To test this hypothesis, we generated a time-pregnant mouse model where wild-type (WT) and Dhcr $7^{+/-}$, carrying WT and Dhcr $7^{+/-}$embryos, respectively, were exposed to ARI or vehicle (VEH) from E12 to E19. The levels of cholesterol, its precursors, ARI, and its metabolites were measured at P0.

\section{Materials and methods}

\section{Chemicals}

Unless otherwise noted, all chemicals were purchased from Sigma-Aldrich Co (St. Louis, MO). High-performance liquid chromatographic-grade solvents were purchased from Thermo Fisher Scientific Inc. (Waltham, MA). Pharmaceutical-grade ARI (marketed as ABILIFY) was obtained from Bristol-Meyers and dissolved in ethanol for the experiments. All sterol standards, natural and isotopically labeled, used in this study are available from Kerafast, Inc. (Boston, MA).

\section{Mouse experiments}

Adult male and female B6.129P2(Cg)-Dhcr $7^{\text {tmlGst }} / \mathrm{J}$ stock \# 007453 mice were purchased from Jackson Laboratories. Mice homozygous for the $D h c r 7^{E x 8}$ allele lack the exon 8 coding sequence and flanking splice acceptor site of the targeted gene, resulting in the truncated DHCR7 mutation most frequently observed in SLOS patients (IVS8-1G>C). Homozygous mice die shortly after birth [38, 39]. Heterozygous $\mathrm{Dhcr} 7^{+/-}$mice are well, fertile, and indistinguishable from control, WT mice. Mice were maintained by breeding within colony and refreshing twice a year with stock 000664 mice from Jackson Laboratories. The mice were housed under a $12 \mathrm{~h}$ light-dark cycle at constant temperature $\left(25^{\circ} \mathrm{C}\right)$ and humidity with ad libitum access to food (Teklad LM-485 Mouse/Rat Irradiated Diet 7912) and water in Comparative Medicine at UNMC, Omaha, NE. The breeding scheme and experimental design is denoted in Fig. 1. The time-pregnant female mice received intraperitoneal injections of VEH or ARI $(5 \mathrm{mg} / \mathrm{kg})$ from E12 to E19. Eight WT and eight Dhcr $7^{+/-}$mothers were used in our study. This exposure window was chosen based on the onset of cholesterol synthesis in the embryonic brain. It has been previously shown that the cholesterol biosynthesis in the brain starts at E12, and from this moment forward, the embryonic brain fully relies on its own cholesterol production [40]. Half of each genotype group was injected with VEH and the other half with ARI. The mouse colony was monitored three times a day and all newborn pups were collected for dissection shortly after the birth. Half of the cortex was used for sterol analysis and the other half was banked for follow-up experiments. Adult female mice were also sacrificed at the same time as pups. Ice-cold lysis buffer (120 mM NaCl, $50 \mathrm{mM}$ HEPES, 1\% Igepal) was added to frozen cortex samples and immediately sonicated. The total protein content was measured using BCA assay (Pierce) and used for normalization. All procedures were performed in accordance with the Guide for the Humane Use and Care of Laboratory Animals. The use of mice in this study was 


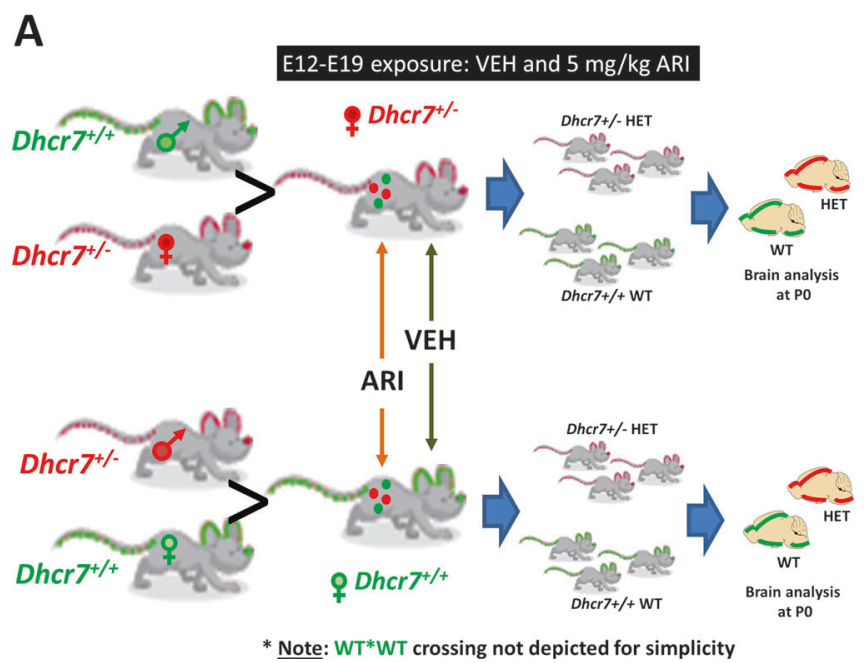

B

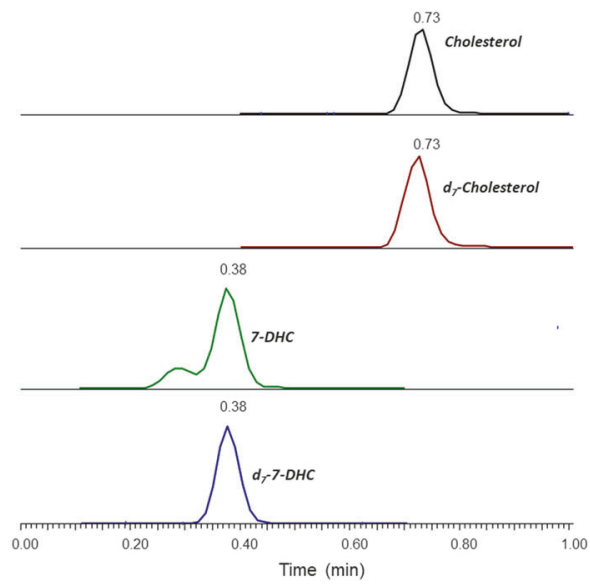

Fig. 1 Experimental design. Dhcr $7^{+/-}$female mice were mated with wild-type (WT) males and $D h c r 7^{+/-}$male mice were mated with WT female mice (a). Pregnant mice were injected with either vehicle or 5 $\mathrm{mg} / \mathrm{kg}$ aripiprazole from E12 to E19. At P0, pups were sacrificed and

approved by the Institutional Animal Care and Use Committee of UNMC.

\section{Liquid chromatography tandem mass spectrometric (selective reaction monitoring (SRM)) analyses}

After lysis, lipids were extracted and derivatized with PTAD as described previously [29] and placed in an Acquity UPLC system equipped with ANSI-compliant well plate holder coupled to a Thermo Scientific TSQ Quantis mass spectrometer equipped with an APCI source. Then 5 $\mu \mathrm{L}$ was injected onto the column (Phenomenex Luna Omega $\mathrm{C} 18,1.6 \mu \mathrm{m}, 100 \AA, 2.1 \times 50 \mathrm{~mm}^{2}$ ) with $100 \%$ $\mathrm{MeOH}(0.1 \% \mathrm{v} / \mathrm{v}$ acetic acid) mobile phase for $1.0 \mathrm{~min}$ runtime at a flow rate of $500 \mu \mathrm{L} / \mathrm{min}$. Natural sterols were analyzed by SRM using the following transitions: Chol $369 \rightarrow 369$, 7-DHC $560 \rightarrow 365$, desmosterol $592 \rightarrow 560$, lanosterol $634 \rightarrow 602$, with retention times of $0.7,0.4,0.3$, and $0.3 \mathrm{~min}$, respectively. SRMs for the internal standards were set to: $\mathrm{d}_{7}$-Chol $376 \rightarrow 376, \mathrm{~d}_{7}-7$-DHC $567 \rightarrow 372$, ${ }^{13} \mathrm{C}_{3}$-desmosterol $595 \rightarrow 563,{ }^{13} \mathrm{C}_{3}$-lanosterol $637 \rightarrow 605$.

Final sterol numbers are reported as $\mathrm{nmol} / \mathrm{mg}$ of protein.

ARI levels were acquired in an Acquity UPLC system coupled to a Thermo Scientific TSQ Quantis mass spectrometer using an ESI source in the positive ion mode. Five $\mu \mathrm{L}$ of each sample was injected onto the column (Phenomenex Luna Omega C18, $1.6 \mu \mathrm{m}, 100 \AA, 2.1 \times 50 \mathrm{~mm}^{2}$ ) using water $(0.1 \% \mathrm{v} / \mathrm{v}$ acetic acid) (solvent $\mathrm{A})$ and acetonitrile $(0.1 \% \mathrm{v} / \mathrm{v}$ acetic acid) (solvent B) as mobile phase. The gradient was: $10-40 \%$ B for $0.5 \mathrm{~min}$; $40-95 \%$ B for $0.4 \mathrm{~min}$; $95 \%$ B for $1.5 \mathrm{~min}$; $95-10 \%$ B for $0.1 \mathrm{~min}$; $10 \% \mathrm{~B}$ for $0.5 \mathrm{~min}$. ARI and its metabolites were analyzed by SRM using the following transitions: ARI $448 \rightarrow 285$, their brain sterol profile was analyzed. b denotes a typical liquid chromatography tandem mass spectrometric chromatogram denoting cholesterol, 7-dehydrocholesterol, and their respective internal standards

dehydroaripiprazole $446 \rightarrow 285$, 2,3-DCPP $230 \rightarrow 187$. The SRM for the internal standards ( $\mathrm{d}_{8}$-ARI and $\mathrm{d}_{8}$ - $\mathrm{mCPP}$ ) were set to $456 \rightarrow 293$ and $204 \rightarrow 157$, respectively. Final drug levels are reported as $\mathrm{ng} / \mathrm{mg}$ of protein.

\section{Statistical analyses}

Statistical analyses were performed using Graphpad Prism 7 for Windows, Microsoft Excel and XLSTAT. Unpaired two-tailed $t$ tests were performed for individual comparisons between two groups. The Welch's correction was employed when the variances between the two groups was significantly different. $p$ Values for statistically significant differences are highlighted in the figure legends. XLSTAT was used to do a comprehensive three-way analysis of variance (ANOVA) to assess the interaction between treatment (VEH vs ARI), maternal genotype (WT vs $D h c r 7^{+-}$) and embryonic genotype (WT vs $D h c r 7^{+-}$). The correlations between drugs were calculated using Pearson's coefficient.

\section{Results}

\section{ARI and its metabolites are detectable in the brains of treated pups}

To investigate the consequences of ARI to the offspring, time-pregnant WT and $D h c r 7^{+/-}$females were injected with either VEH or $5 \mathrm{mg} / \mathrm{kg}$ ARI. To confirm that maternal ARI exposure resulted in placental drug transfer to the embryos, we measured ARI and its metabolites in the brain of all tested pups. While none of the compounds were detected in 


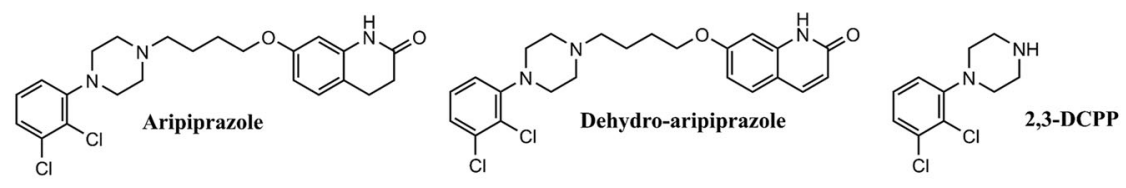

A

Aripiprazole vs Dehydroaripiprazole

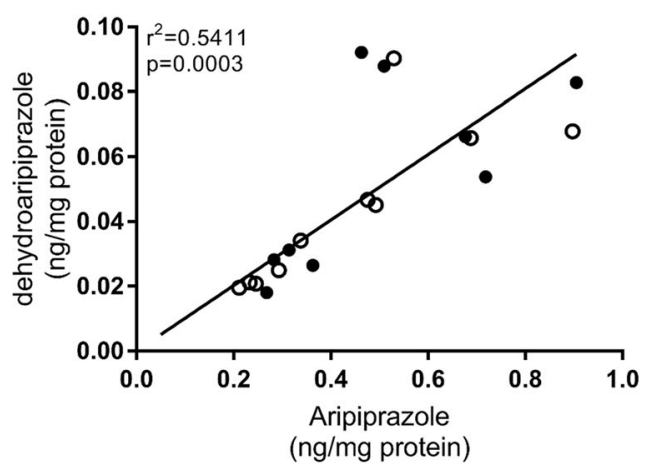

Fig. 2 Aripiprazole (ARI) and its metabolites cross the placental barrier. ARI and its metabolites were readily detectable in the brain of newborn pups. A positive correlation between ARI and dehydroaripiprazole (a) and 2,3-dichlorophenylpiperazine (b) was observed in the analyzed tissue. Only ARI-treated pup data are disclosed, as in vehicle-treated pup brains no ARI or metabolites were detected. Each

the VEH-injected animals, ARI and its metabolitesdehydroaripiprazole and 1-(2,3-dichlorophenyl)piperazine (2,3-DCPP) -were detected in all brain samples from the ARI-injected group (Fig. 2). Importantly, we found a positive correlation between ARI and its metabolites in the brain of pups, regardless of maternal or embryonic genotype. This experiment confirmed that ARI was transported through the placenta, reached the brain of embryos, and had the potential to alter the sterol metabolism of the developing brain in utero.

\section{ARI decreases litter size}

As an initial assessment of ARI's impact on embryonic development, we examined the effect of ARI on the litter size and pups per litter. Table 1 shows the number of mothers used in each experimental group, number of pups delivered from each group, and the pups/mother ratio. The litter sizes from animals injected with ARI were substantially smaller when compared to those from animals injected with VEH: 57 pups were delivered from 8 females injected with VEH, while only 37 were delivered from the same number of females injected with ARI, representing a $35 \%$ reduction in the litter size $(p<0.05)$. This was also evidenced when the pups/mother ratio is analyzed, which was smaller in the groups that received ARI injection (VEH: 7.1 and ARI: 4.6 pups/mother). Moreover, while 3 stillborn pups were identified in the ARI-injected group, none were observed in the VEH-injected group. Interestingly, the
B

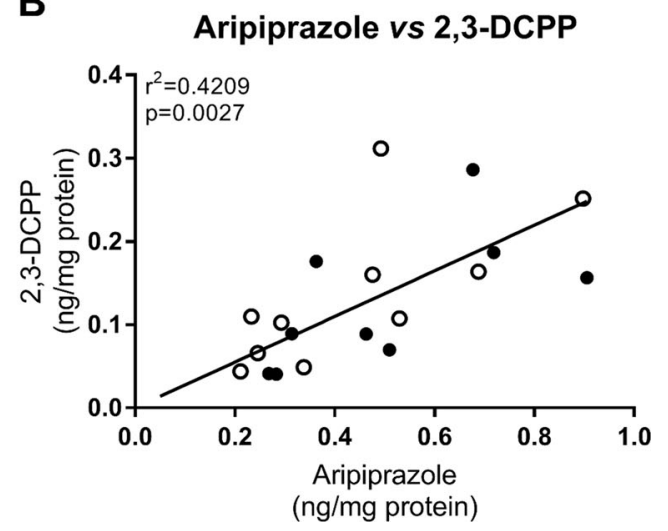

symbol denotes ARI level in a single ARI-treated brain sample $(n=$ 19), filled symbols denote pups with a $D h c r 7^{+/+}$genotype (wild type), open symbols denote pups with a $D h c r 7^{+/-}$genotype. Pearson correlation coefficients ( $r^{2}$ and $p$ values) were calculated using GraphPad Prism 7

Table 1 Viability of VEH- and ARI-treated pregnancies

\begin{tabular}{lllll}
\hline Treatment & Female $(n)^{\mathrm{a}}$ & Pups & \\
\cline { 3 - 5 } & & Total & $\left(\right.$ Dhcr $\left.7^{+/+}\right)$ & $\left(\right.$Dhcr $\left.7^{+/}\right)$ \\
\hline Vehicle & Dhcr $^{+/+}(4)$ & 26 & 15 & 11 \\
& Dhcr $^{+/-}(4)$ & 31 & 14 & 17 \\
Aripiprazole $^{\mathrm{b}}$ & Dhcr $^{+/+}(4)$ & 17 & 8 & 9 \\
& $D h c r 7^{+/-}(4)$ & 20 & 10 & 10 \\
\hline
\end{tabular}

All VEH vs All ARI $-p<0.05$

All $D h c r 7^{+/+}$vs All $D h c r 7^{+/-}$—non-significant

VEH: $D h c r 7^{+/+}$vs $D h c r 7^{+/-}$-non-significant

ARI: $D h c r 7^{+/+}$vs $D h c r 7^{+/-}$-non-significant

ARI aripiprazole, $V E H$ vehicle

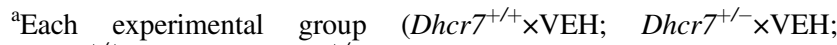
Dhcr $7^{+/+} \times$ARI and $D h c r 7^{+/-} \times$ARI) consisted of four pregnant mice. Note that the ARI-treated litters were smaller $\left(D h c r 7^{+/+}-17\right.$ pups; Dhcr $7^{+/-}-20$ pups) than the VEH-treated litters (Dhcr $7^{+/+}-26$ pups, Dhcr $7^{+/-}-31$ pups). Furthermore, ARI-treated litters resulted in three stillborn pregnancies. Note also that the pups/litter ratio is $35 \%$ smaller in the ARI-injected group (VEH: 7.1 pups/litter and ARI: 4.6 pups/ litter). Of all the comparisons, only the VEH vs ARI pups/litter reached significance at $p<0.05$. In addition, the sex of the pups did not differ between any of the compared groups

${ }^{\mathrm{b}}$ Females were injected daily with either vehicle or $5 \mathrm{mg} / \mathrm{kg}$ aripiprazole from E12 to E19

mothers' genotype $\left(D h c r 7^{+/-}\right.$or $\left.D h c r 7^{+/+}\right)$had no apparent effect on these findings. Overall, these observations show that ARI has a considerable effect on offspring viability. While the smaller litters are clearly not a result of altered 
A
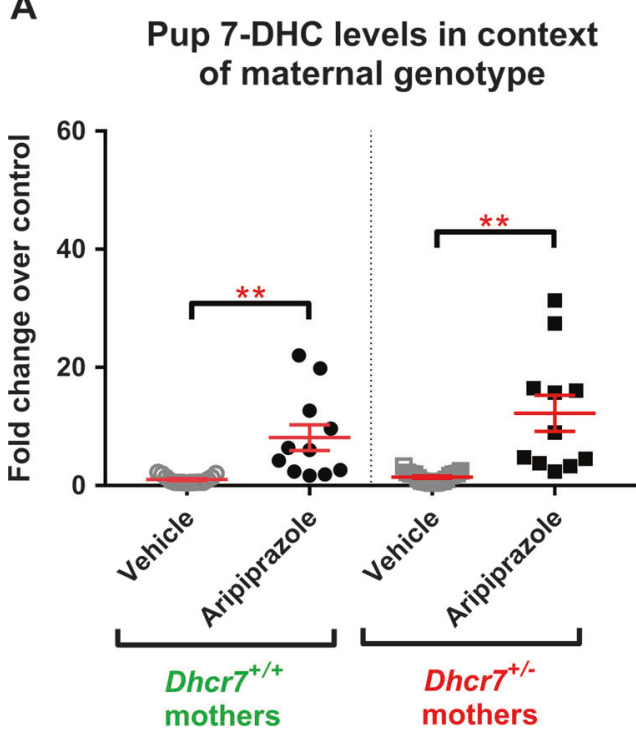

Fig. 3 7-Dehydrocholesterol (7-DHC) levels in the P0 brain of pups maternally exposed to vehicle or aripiprazole (ARI) $(5 \mathrm{mg} / \mathrm{kg}$ ). a shows changes in 7-DHC in the context of the maternal genotype. Both wild-type (WT) and $D h c r 7^{+/-}$pups were grouped taking into account only their mothers' genotype. b shows changes in 7-DHC in the context of the embryonic genotype. Pups were grouped taking into account only their own genotypes, regardless if they were born from WT or $D h c r 7^{+/-}$mothers. Note that 7-DHC is increased as a result of ARI injection and it is dependent both on the maternal (a) and

fertilization of embryonic implantation, we were unable the precisely determine the precise timing of the premature demise. Perhaps the three stillborn pups suggest that ARI exposure is likely to affect the later stages of pregnancy, but this should be further investigated. It is also noteworthy that we did not observe differences in the ratio of male-tofemale pups in any of our treatment groups, suggesting that the effects of ARI are not preferentially affecting one of the sexes.

\section{Pups born to Dhcr $7^{+/-}$mothers show increased vulnerability to ARI exposure}

Embryonic development is a period of active sterol biosynthesis in the brain [1,2]. To assess the consequences of ARI treatment on the sterol profile during this period, we analyzed the brains of the pups immediately after birth (P0). To answer the question whether the maternal and embryonic genotypes affect the 7-DHC response to ARI, P0 pups were grouped and analyzed according to their mothers' (Fig. 3a) or their own genotypes (Fig. 3b). 7-DHC values were normalized and are reported as fold change over control using VEH-injected pups from the WT group as the control condition (i.e., WT-vehicle $=1$ ). We found that ARI induced a 12-fold increase in 7-DHC in the pups born to $D h c r 7^{+/-}$mothers, compared to an 8-fold increase
B

\section{Pup 7-DHC levels in context of embryonic genotype}

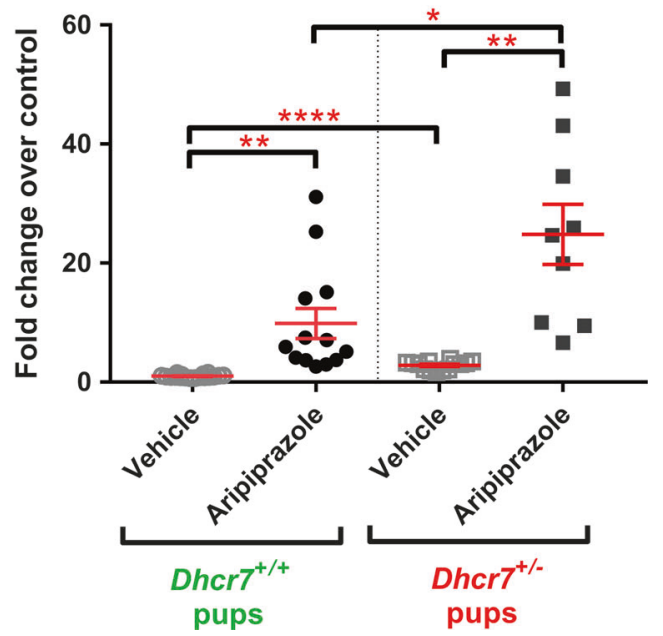

embryonic genotypes (b). Values were normalized against the control condition (i.e., WT-Vehicle $=1$ ). The genotypes are depicted below the groups; Statistical significance: $* p<0.05 ; * * p<0.01 ; * * * * p<$ 0.0001. Bars correspond to the mean \pm SEM. Each symbol corresponds to a single pup brain. Note that $D h c r 7^{+/-}$pups have higher basal 7-DHC levels compared to their WT littermates (comparison between vehicle conditions in $\mathbf{b}$ ), which becomes more pronounced in ARI-injected group. The raw sterol values are reported in Supplemental Material 1

in 7-DHC that was observed in mice from WT mothers (Fig. 3a). The more pronounced 7-DHC elevation in the brain of pups from $D h c r 7^{+/-}$mothers suggests that $D h c r 7^{+/-}$maternal genotype increases the vulnerability of pups to ARI exposure. Absolute 7-DHC levels are reported in Figure S1 in the supporting information. Levels of cholesterol (Figure S2), desmosterol (Figure S3), and lanosterol (Figure S4) were much less significantly changed and are included in the supporting information.

\section{ARI has the most pronounced biochemical effect on the brain of $\mathrm{DhCr}^{+l-}$ pups}

We hypothesized that maternal ARI exposure would have the largest effect on 7-DHC in the brain of $\mathrm{Dhcr}^{+/-}$pups. Figure $3 \mathrm{~b}$ denotes the changes in 7-DHC in response to ARI injection in the context of the embryonic Dhcr 7 genotype. Brain samples from WT pups injected with VEH had the lowest 7-DHC levels among all groups. $D h c r 7^{+/-}$pups had higher basal 7-DHC levels than their WT littermates, further suggesting mutations in one Dhcr 7 allele alone leads to an altered biochemical phenotype (comparison between VEH-exposed conditions). ARI increased 7-DHC levels in both WT and $D h c r 7^{+/-}$ pups when compared to the VEH-injected groups, which confirms that ARI increases 7-DHC in utero. However, as 
A

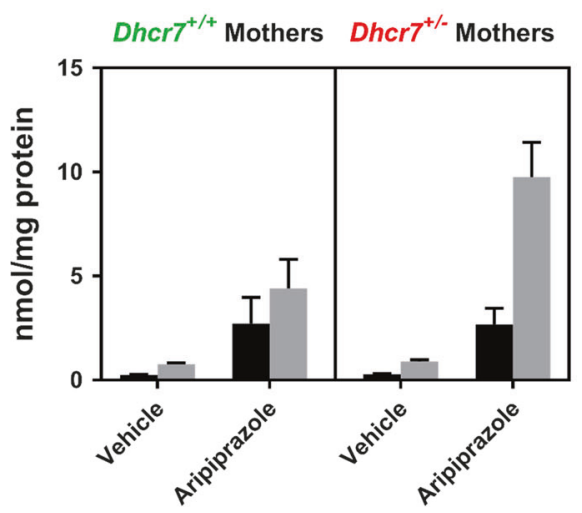

Fig. 4 7-Dehydrocholesterol (7-DHC) levels found in the brain of P0 pups exposed to vehicle or aripiprazole $(5 \mathrm{mg} / \mathrm{kg})$. 7-DHC levels (a) and 7-DHC/cholesterol ratio (b) are grouped taking into account all the three variables: maternal genotype, embryonic genotype, and drug treatment (three-way analysis). Black and gray columns denote wildtype and $D h c r 7^{+-}$pups, respectively. The mothers' genotypes are

hypothesized, $D h c r 7^{+/-}$pups were the most vulnerable to ARI's effects and reported even higher 7-DHC levels than their WT littermates subjected to the same ARI exposure: while ARI injection resulted in a 10-fold increase in pups with a WT genotype, the same ARI exposure resulted in an even more remarkable, 25-fold increase in 7-DHC levels in pups with a $D h c r 7^{+/-}$genotype. These results indicate that a $D h c r 7^{+-}$embryonic genotype also increases the vulnerability to ARI exposure. Altogether, the two panels of Fig. 3 clearly indicate that both maternal and embryonic Dhcr 7 genotypes are determinants of how the developing brain responds to ARI.

\section{Maternal genotypexembryonic genotypexARI treatment interaction}

In the next step, we tested the hypothesis that the combination of both maternal and embryonic $D h c r 7^{+/-}$genotypes would alter ARI's effect on 7-DHC, which would result in the highest and most toxic levels of 7-DHC. To address that, we did a three-way ANOVA analysis of 7-DHC levels in the brain of pups taking into account the maternal genotype (WT or $D h c r 7^{+/-}$), the embryonic genotype (WT or $D h c r 7^{+/-}$), and treatment (VEH or ARI). These results are presented in Fig. 4a and Table 2. As hypothesized, $D h c r 7^{+/-}$pups from $D h c r 7^{+/-}$mothers were the most vulnerable to ARI and presented the highest levels of 7-DHC. Figure 4a also shows that in all conditions $D h c r 7^{+-}$pups had higher 7-DHC levels than their WT littermates. A comprehensive analysis of these results (Table 2) reveals that: (1) a combination of maternal and embryonic $D h c r 7^{+/-}$genotypes have a significant impact on 7-DHC; (2) ARI interacts with the maternal $\mathrm{Dhcr}^{+/-}$genotype and potentiates the effect on

\section{7-DHC/cholesterol in PO Brain}

Dher ${ }^{+/+}$Mothers Dhcr $^{+/-}$Mothers

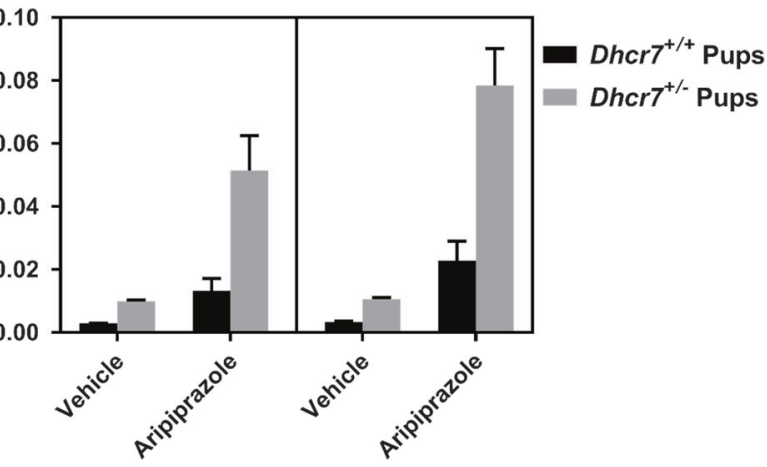

depicted above the graph. Values correspond to the mean \pm SEM. Note that the 7-DHC levels are the highest in $D h c r 7^{+/-}$pups from $D h c r 7^{+/-}$ mothers, indicating a synergism between maternal genotypexembryonic genotypextreatment. Comprehensive statistical analysis of data is presented in Table 2

Table 2 Effect of three variables on 7-DHC levels in the pup brain

\begin{tabular}{|c|c|c|}
\hline No. & Comparison & $p$ Value \\
\hline 1 & Treatment effect: ARI vs VEH & $<0.0001$ \\
\hline 2 & Mother genotype effect: $D h c r 7^{+/-}$vs Dhcr $7^{+/+}$ & 0.010 \\
\hline 3 & Pup genotype effect: $D h c r 7^{+/-}$vs $D h c r 7^{+/+}$ & $<0.0001$ \\
\hline 4 & $\begin{array}{l}\text { Two-way interaction: Treatment (ARI, } \\
\text { VEH) } \times \text { Maternal } D h c r 7 \text { genotype }\left({ }^{+/} \text {or }{ }^{+/+}\right)\end{array}$ & 0.015 \\
\hline 5 & $\begin{array}{l}\text { Two-way interaction: Treatment (ARI, VEH) } \times \text { Pup } \\
\text { Dhcr } 7 \text { genotype }\left({ }^{+/-} \text {or }{ }^{+/+}\right)\end{array}$ & $<0.000$ \\
\hline 6 & $\begin{array}{l}\text { Two-way interaction: Maternal } D h c r 7 \text { genotypexPup } \\
\text { Dhcr7 genotype }\end{array}$ & 0.010 \\
\hline 7 & $\begin{array}{l}\text { Three-way interaction: Treatment } \times \text { Maternal } \text { Dhcr } 7 \\
\text { genotypexPup Dhcr } 7 \text { genotype }\end{array}$ & 0.013 \\
\hline
\end{tabular}

The table represents the outcome of multifactorial analysis of variance model progressively accounting for all three variables we tested: treatment (ARI, VEH), pup Dhcr 7 genotype $(+/-$ vs $+/+)$, and maternal Dhcr7 genotype $(+/-$ vs $+/+)$. Rows $1-3$ denote significance for each of the single variable; row 3-6 reports probability for two of the interacting factors; row 7 denotes interaction between all the three tested variables

$A R I$ aripiprazole, 7-DHC 7-dehydrocholesterol, $V E H$ vehicle

7-DHC; (3) ARI interacts with the embryonic Dhcr $7^{+/}$ genotype and potentiates the effect on 7-DHC; (4) the most significant effect on 7-DHC comes from ARI treatment, which is further affected by both maternal and embryonic $D h c r 7^{+/-}$genotypes. Altogether, these results suggest that $D h c r 7^{+-}$pups from $D h c r 7^{+/-}$mothers are highly vulnerable to ARI effects.

Notably, these data are also concordant with our previously published data on the effect of ARI-treated $\mathrm{DHCR}^{+/+}$and $D H C R 7^{+/-}$human dermal fibroblasts [22], suggesting that the DHCR7 genotypexARI treatment interaction is not a 
A

Total pup DHC/cholesterol ratio in context of maternal genotype

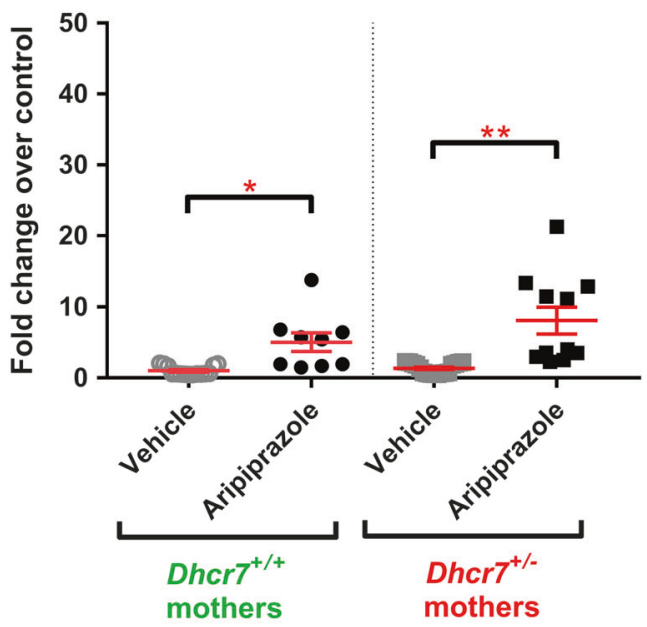

Fig. 5 7-Dehydrocholesterol (7-DHC)/cholesterol ratio in the brain of P0 pups exposed to vehicle or aripiprazole (ARI; $5 \mathrm{mg} / \mathrm{kg}$ ). a shows changes in 7-DHC/cholesterol in the context of the maternal genotype. Both wild-type (WT) and $\mathrm{Dhcr} 7^{+/-}$pups were grouped taking into account only their mothers' genotype. b shows changes in 7-DHC/ cholesterol in the context of the embryonic genotype. Pups were grouped taking into account only their own genotypes, regardless if they were born from WT or Dhcr $7^{+/-}$mothers. 7-DHC/cholesterol ratio is increased as a result of ARI injection and it is dependent on the

mouse-specific physiological event, thus underscoring the potential clinical implications of our findings.

\section{ARI-induced alterations in the 7-DHC/cholesterol ratio are $D h c r 7$ genotype-dependent}

In addition to the measurement of 7-DHC levels, we also assessed cholesterol biosynthesis, which can be measured by the 7-DHC/cholesterol ratio [22, 29]. Under control conditions, where cholesterol biosynthesis is functioning perfectly and the DHCR7 enzyme is operating properly, the 7-DHC/cholesterol ratio is extremely small (see Figure S5 in the supporting information for the "absolute" ratio values for all groups). DHCR7 inhibition leads to higher 7-DHC and lower cholesterol levels and therefore a higher 7-DHC/ cholesterol ratio (Fig. 5), which can be used as a dynamic readout of the health of the sterol synthesis system.

Figure 5a denotes the ARI-induced changes in the 7DHC/cholesterol ratio accounting for the mothers' Dhcr 7 genotype. ARI induced a five-fold increase in the 7-DHC/ cholesterol ratio in pups from WT mothers, while the same treatment lead to an eight-fold increase in the 7-DHC/cholesterol ratio in pups from $D h c r 7^{+/-}$mothers (Fig. 5a). These observations further suggest that a $D h c r 7^{+/-}$maternal genotype leads to an imbalance between cholesterol and its precursor.

\section{B \\ Total pup $\mathrm{DHC} / \mathrm{cholesterol} \mathrm{ratio}$ in context of embryonic genotype}

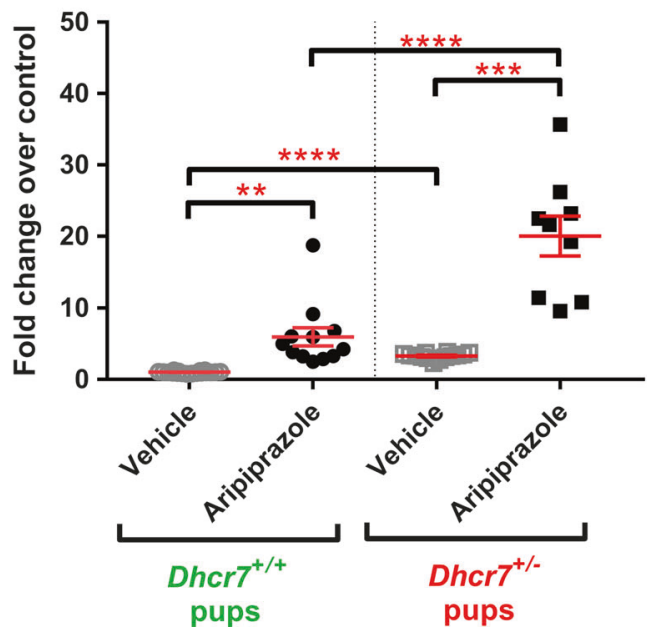

maternal (a) and embryonic genotypes (b). Values were normalized against the control condition (i.e. WT-Vehicle $=1$ ). The genotypes are depicted below the groups. Statistical significance: ${ }^{*} p<0.05$; ${ }^{* *} p<$ $0.01 ; * * * p<0.001 ; * * * * p<0.0001$. Bars correspond to the mean \pm SEM. Each symbol corresponds to a single pup. Note that the 7-DHC/ cholesterol ratio in $\mathrm{Dhcr}^{+/-}$pups is elevated compared to their WT littermates (b). This difference is enhanced in the ARI-injected group, highlighting increased vulnerability of $\mathrm{Dhcr} 7^{+/-}$to ARI's side effects. The raw sterol ratio values are reported in supporting information

ARI's effects on the 7-DHC/cholesterol ratio in the context of pups' genotype are shown in Fig. 5b. As observed previously for 7-DHC, the embryonic $\mathrm{Dhcr} \mathrm{7}^{+/-}$genotype had a pronounced effect both on the basal 7-DHC/cholesterol ratio and its response to ARI injection. Compared to the VEHinjected pups, the 7-DHC/cholesterol ratio was also significantly elevated in the ARI-injected pups. Indeed, while ARI injection increased the 7-DHC/cholesterol ratio by 6-fold in WT pups, the same treatment resulted in a 20 -fold increase in the $D h c r 7^{+/-}$pup group, highlighting an imbalance of the sterol biosynthesis pathway.

An analysis of the 7-DHC/cholesterol ratio taking into account the maternal genotype, embryonic genotype, and treatment (three-way analysis) is presented in Fig. 4b. The 7DHC/cholesterol ratio responded in a similar way as observed for 7-DHC in Fig. 4a, where the highest 7-DHC/cholesterol ratio is observed in $D h c r 7^{+/-}$pups from $\mathrm{Dhcr} 7^{+/-}$mothers injected with ARI. These results provide further evidence for a strong interaction between maternal Dhcr7 genotypexembryonic Dhcr 7 genotype $\times A R I$ treatment.

\section{Discussion}

Normal cholesterol metabolism is of critical importance for neurodevelopment. Once the blood-brain barrier is closed, 
which happens during embryonic development, the brain has to synthesize its own cholesterol $[2,4,41]$. The importance of a normal sterol metabolism is evidenced by the many genetic disorders associated with mutations in cholesterol biosynthesis enzymes [5-8]. Therefore, a chemical interference with this biosynthetic pathway could have profound effects on the development and health of the offspring.

ARI has been used to treat patients with schizophrenia and bipolar disorders for many years and its beneficial effects to patients are well known [42, 43]. Side effects of ARI have been extensively documented, including the modulation of the cholesterol biosynthesis both in vitro and in vivo [23, 28, 42], but relatively little attention has been paid to these processes in the context of pregnancy, maternal and offspring genotype, and potential pathological changes in the offspring.

Our results show that maternal exposure to ARI is potentially deleterious on embryonic development. There are eight major conclusions that we can draw from our study. (1) ARI and its metabolites are transported across the placenta and reach the brain of fetuses during embryonic development. (2) VEH-treated $D h c r 7^{+/-}$pups have higher than normal 7-DHC levels than their WT littermates, which is in concordance with observations in adult humans carrying the $\mathrm{DHCR} 7^{+/-}$genotype. (3) Maternal ARI exposure leads to decreased viability of embryos, as evidenced by reduced litter size. (4) ARI inhibits the DHCR7 enzyme in the brain of all embryos and leads to increased 7-DHC levels, regardless of maternal or offspring Dhcr 7 genotypes. (5) ARI increases 7-DHC levels in WT pups to levels higher than those observed in $\mathrm{Dhcr}^{+/-}$pups under VEH-treated conditions. (6) Based on the sterol biosynthesis profile, $D h c r 7^{+/-}$pups are more vulnerable to maternal ARI exposure than their WT littermates. This drug $\times$ genotype interaction might lead to a chemically induced SLOS phenotype, which should be further investigated. (7) There is a significant difference between the response of the pups from WT and $D h c r 7^{+/-}$mothers to maternal ARI exposure, and we can conclude that the mothers' genotype also influences the pups' response to the treatments and increases their vulnerability to cholesterol biosynthesis inhibitors. (8) Based on 7-DHC level measurements, maternal $\mathrm{Dhcr}^{+/-}$ genotype, embryonic $D h c r 7^{+/-}$genotype, and ARI treatment interact and potentiate each other's effects. This suggests that both mothers' and pups' genotype carry the vulnerability to ARI exposure. This confirms our initial hypothesis that the strongest effects of maternal ARI exposure would be observed in $D h c r 7^{+/-}$pups originating from $D h \mathrm{cr}^{+/-}$pregnant dams. This simultaneously highlights that paternally inherited $\mathrm{DHCR} 7^{+/-}$genotype combined with ARI exposure represents a risk, even when the mother is $\mathrm{DHCR} 7^{+/+}$. This should be examined in followup studies.
So, the first critical question becomes: what are the overall biological consequences of 7-DHC elevation and disruption of sterol biosynthesis observed in our studies? Our study did not assess the gross anatomical and microanatomical brain changes in the ARI-exposed pups or attempted behavioral assessments. Based on changes in the brains of patients with SLOS and animal models of the disorder, we can only hypothesize at this time that observed biochemical impact would give rise to alteration in the serotonergic system [44] and corpus callosum connectivity [45], and this should be further investigated. Still, several recent publications indicate that our findings should not be taken lightly. Recently, Bolland and Tatonetti comprehensively reviewed the effects of cholesterol-altering drugs on pregnancy outcomes in humans [46] and linked inhibitors of cholesterol biosynthesis to a wide range of negative pregnancy outcomes. Importantly, they found that antipsychotics, such as clozapine and haloperidol and cholesterol-lowering drugs (statins), were among those with the most deleterious effects on fetal development. Notably, these findings did not account for a $D H C R 7$ genotype as a variable that might further influence the outcome. Thus these findings suggest that any pharmacologically active substance should be closely examined for its potential to interfere with offspring cholesterol biosynthesis during pregnancy, and perhaps it is worth considering a dual parental and fetal DHCR7 genotyping before prescribing such medications.

If we accept that there are potential biological consequences of unwanted 7-DHC elevation, it leads us to the second question: should pharmacological compounds with effects on cholesterol biosynthesis be prescribed to $D H C R 7^{+/-}$mutation carrier children and adults? The answer to this question is less clear: while $D H C R 7^{+/-}$ mutation carriers have elevated baseline DHC levels, they are generally considered healthy, and there is no current data that speak of drugxgenotype interactions in these individuals. Clearly, this could be examined in epidemiological genome-wide association studies. However, it is clear that individuals with SLOS, who already carry two mutant DHCR7 alleles and have remarkable elevations of 7-DHC, should not be prescribed ARI and other medications that act as cholesterol biosynthesis inhibitors.

In conclusion, considering that ARI is a very commonly used medication, often prescribed to pregnant women, we believe that there are important clinical implications of our study. (1) SLOS patients should clearly avoid drugs that increase 7-DHC levels, as they have already extremely high, toxic levels of 7-DHC, and any further increase might be detrimental. (2) Treatment with 7-DHC-elevating substances (such as ARI, trazodone, and haloperidol) might be potentially unsafe for the $1-1.5 \%$ of population with singleallele disruptions of the $D H C R 7$ gene. (3) Vulnerability to 
7-DHC-elevating compounds appears to be most pronounced during pregnancy and brain development, and both prenatal and parental genetic testing for DHCR7 should be considered before prescribing sterol-interfering medications during pregnancy. Such testing or a choice of a different medication could avoid a chemically induced SLOS phenotype in offspring who carries a single allele disruption of the DHCR7 gene. Finally, it should be stressed that ARI (and many other medications affecting sterol biosynthesis) are safe and life-saving medications for $99 \%$ of the population, but in this era of precision/personalized medicine, we must recognize the potential vulnerability of a subpopulation of our patients to these pharmacological compounds. After all, this maternal genotypexembryonic genotypextreatment interaction is a cornerstone of personalized medicine and moves us from the primum non nocere to the primum non nocere et optimum curare concept.

Acknowledgements This work was supported by The National Institutes of Health, NICHD HD064727 (to NAP), NIMH MH110636 (to $\mathrm{KM}$ ), and MN067234 (to KM).

Author contributions Study design: KM, NAP, and ZK; funding: NAP, KM; syntheses of standards: KAT; LC-MS/MS analysis: KAT, TCG-M; mouse colony maintenance, treatment, and dissection: AA, LBA, and ZK; mouse sample preparation, sterol extraction: LBA, ZK, TCG-M; statistical analysis: TCG-M; draft of manuscript: TCG-M, $\mathrm{ZK}$, and $\mathrm{KM}$; final version read and approved by all authors.

\section{Compliance with ethical standards}

Conflict of interest The authors declare that they have no conflict of interest.

Publisher's note: Springer Nature remains neutral with regard to jurisdictional claims in published maps and institutional affiliations.

Open Access This article is licensed under a Creative Commons Attribution 4.0 International License, which permits use, sharing, adaptation, distribution and reproduction in any medium or format, as long as you give appropriate credit to the original author(s) and the source, provide a link to the Creative Commons license, and indicate if changes were made. The images or other third party material in this article are included in the article's Creative Commons license, unless indicated otherwise in a credit line to the material. If material is not included in the article's Creative Commons license and your intended use is not permitted by statutory regulation or exceeds the permitted use, you will need to obtain permission directly from the copyright holder. To view a copy of this license, visit http://creativecommons. org/licenses/by/4.0/.

\section{References}

1. Dietschy JM, Turley SD. Cholesterol metabolism in the brain. Curr Opin Lipidol. 2001;12:105-12.

2. Dietschy JM, Turley SD. Thematic review series: brain lipids. Cholesterol metabolism in the central nervous system during early development and in the mature animal. J Lipid Res. 2004;45:1375-97.
3. Bjorkhem I. Crossing the barrier: oxysterols as cholesterol transporters and metabolic modulators in the brain. J Intern Med. 2006;260:493-508.

4. Nes WD. Biosynthesis of cholesterol and other sterols. Chem Rev. 2011;111:6423-51.

5. Herman GE. Disorders of cholesterol biosynthesis: prototypic metabolic malformation syndromes. Hum Mol Genet. 2003;12 Spec No.1:R75-88.

6. Nwokoro NA, Wassif CA, Porter FD. Genetic disorders of cholesterol biosynthesis in mice and humans. Mol Genet Metab. 2001;74:105-19.

7. Porter FD. RSH/Smith-Lemli-Opitz syndrome: a multiple congenital anomaly/mental retardation syndrome due to an inborn error of cholesterol biosynthesis. Mol Genet Metab. 2000;71:16374.

8. Porter FD, Herman GE. Malformation syndromes caused by disorders of cholesterol synthesis. J Lipid Res. 2011;52:6-34.

9. Smith DW, Lemli L, Opitz JM. A newly recognized syndrome of multiple congenital anomalies. J Pediatr. 1964;64:210-7.

10. Korade Z, Xu L, Mirnics K, Porter NA. Lipid biomarkers of oxidative stress in a genetic mouse model of Smith-Lemli-Opitz syndrome. J Inherit Metab Dis. 2012;36:113-22.

11. Jira PE, Waterham HR, Wanders RJ, Smeitink JA, Sengers RC, Wevers RA. Smith-Lemli-Opitz syndrome and the DHCR7 gene. Ann Hum Genet. 2003;67:269-80.

12. Kelley RI, Hennekam RC. The Smith-Lemli-Opitz syndrome. J Med Genet. 2000;37:321-35.

13. Windsor K, Genaro-Mattos TC, Kim HY, Liu W, Tallman KA, Miyamoto S, et al. Probing lipid-protein adduction with alkynyl surrogates: application to Smith-Lemli-Opitz syndrome. J Lipid Res. 2013;54:2842-50.

14. Xu L, Mirnics K, Bowman AB, Liu W, Da J, Porter NA, et al. DHCEO accumulation is a critical mediator of pathophysiology in a Smith-Lemli-Opitz syndrome model. Neurobiol Dis. 2011;45:923-9.

15. Xu L, Korade Z, Rosado DA Jr., Liu W, Lamberson CR, Porter NA. An oxysterol biomarker for 7-dehydrocholesterol oxidation in cell/mouse models for Smith-Lemli-Opitz syndrome. J Lipid Res. 2011;52:1222-33.

16. Xu L, Davis TA, Porter NA. Rate constants for peroxidation of polyunsaturated fatty acids and sterols in solution and in liposomes. J Am Chem Soc. 2009;131:13037-44.

17. Yin $\mathrm{H}, \mathrm{Xu} \mathrm{L}$, Porter NA. Free radical lipid peroxidation: mechanisms and analysis. Chem Rev. 2011;111:5944-72.

18. Xu L, Korade Z, Rosado DA Jr., Mirnics K, Porter NA. Metabolism of oxysterols derived from nonenzymatic oxidation of 7dehydrocholesterol in cells. J Lipid Res. 2013;54:1135-43.

19. Korade Z, Xu L, Shelton R, Porter NA. Biological activities of 7dehydrocholesterol-derived oxysterols: implications for SmithLemli-Opitz syndrome. J Lipid Res. 2010;51:3259-69.

20. Nowaczyk MJ, Nakamura LM, Eng B, Porter FD, Waye JS. Frequency and ethnic distribution of the common DHCR7 mutation in Smith-Lemli-Opitz syndrome. Am J Med Genet. 2001;102:383-6.

21. Cross JL, Iben J, Simpson CL, Thurm A, Swedo S, Tierney E, et al. Determination of the allelic frequency in Smith-Lemli-Opitz syndrome by analysis of massively parallel sequencing data sets. Clin Genet. 2015;87:570-5.

22. Korade Z, Genaro-Mattos TC, Tallman KA, Liu W, Garbett KA, Koczok K, et al. Vulnerability of DHCR7 $(+/-)$ mutation carriers to aripiprazole and trazodone exposure. J Lipid Res. 2017;58:2139-46.

23. Hall P, Michels V, Gavrilov D, Matern D, Oglesbee D, Raymond $\mathrm{K}$, et al. Aripiprazole and trazodone cause elevations of 7dehydrocholesterol in the absence of Smith-Lemli-Opitz Syndrome. Mol Genet Metab. 2013;110:176-8. 
24. Kim HY, Korade Z, Tallman KA, Liu W, Weaver CD, Mirnics K, et al. Inhibitors of 7-dehydrocholesterol reductase: screening of a collection of pharmacologically active compounds in Neuro2a cells. Chem Res Toxicol. 2016;29:892-900.

25. Chambers CM, McLean MP, Ness GC. Smith-Lemli-Opitz syndrome produced in rats with AY 9944 treated by intravenous injection of lipoprotein cholesterol. Am J Med Genet. 1997;68:322-7.

26. Giera M, Plossl F, Bracher F. Fast and easy in vitro screening assay for cholesterol biosynthesis inhibitors in the post-squalene pathway. Steroids. 2007;72:633-42.

27. Canfran-Duque A, Casado ME, Pastor O, Sanchez-Wandelmer J, de la Pena G, Lerma M, et al. Atypical antipsychotics alter cholesterol and fatty acid metabolism in vitro. J Lipid Res. 2013;54:310-24.

28. Korade Z, Liu W, Warren EB, Armstrong K, Porter NA, Konradi C. Effect of psychotropic drug treatment on sterol metabolism. Schizophr Res. 2017;187:74-81.

29. Genaro-Mattos TC, Tallman KA, Allen LB, Anderson A, Mirnics $\mathrm{K}$, Korade $\mathrm{Z}$, et al. Dichlorophenyl piperazines, including a recently-approved atypical antipsychotic, are potent inhibitors of DHCR7, the last enzyme in cholesterol biosynthesis. Toxicol Appl Pharmacol. 2018;349:21-28.

30. Gentile S, Tofani S, Bellantuono C. Aripiprazole and pregnancy: a case report and literature review. J Clin Psychopharmacol. 2011;31:531-2.

31. Gentile S. A safety evaluation of aripiprazole for treating schizophrenia during pregnancy and puerperium. Expert Opin Drug Saf. 2014;13:1733-42.

32. Lutz UC, Hiemke C, Wiatr G, Farger G, Arand J, Wildgruber D. Aripiprazole in pregnancy and lactation: a case report. J Clin Psychopharmacol. 2010;30:204-5.

33. Mendhekar DN, Sharma JB, Srilakshmi P. Use of aripiprazole during late pregnancy in a woman with psychotic illness. Ann Pharmacother. 2006;40:575.

34. Mervak B, Collins J, Valenstein M. Case report of aripiprazole usage during pregnancy. Arch Womens Ment Health. 2008;11:249-50.
35. Widschwendter CG, Hofer A. Aripiprazole use in early pregnancy: a case report. Pharmacopsychiatry. 2012;45:299-300.

36. Alavijeh MS, Chishty M, Qaiser MZ, Palmer AM. Drug metabolism and pharmacokinetics, the blood-brain barrier, and central nervous system drug discovery. NeuroRx. 2005;2:554-71.

37. Wang JS, Zhu HJ, Donovan JL, Yuan HJ, Markowitz JS, Geesey $\mathrm{ME}$, et al. Aripiprazole brain concentration is altered in Pglycoprotein deficient mice. Schizophr Res. 2009;110:90-4.

38. Fitzky BU, Moebius FF, Asaoka H, Waage-Baudet H, Xu L, Xu G, et al. 7-Dehydrocholesterol-dependent proteolysis of HMG-CoA reductase suppresses sterol biosynthesis in a mouse model of SmithLemli-Opitz/RSH syndrome. J Clin Invest. 2001;108:905-15.

39. Wassif CA, Zhu P, Kratz L, Krakowiak PA, Battaile KP, Weight $\mathrm{FF}$, et al. Biochemical, phenotypic and neurophysiological characterization of a genetic mouse model of RSH/Smith-Lemli-Opitz syndrome. Hum Mol Genet. 2001;10:555-64.

40. Tint GS, Yu H, Shang Q, Xu G, Patel SB. The use of the Dhcr7 knockout mouse to accurately determine the origin of fetal sterols. J Lipid Res. 2006;47:1535-41.

41. Obermeier B, Daneman R, Ransohoff RM. Development, maintenance and disruption of the blood-brain barrier. Nat Med. 2013;19:1584-96.

42. Citrome L. A review of aripiprazole in the treatment of patients with schizophrenia or bipolar I disorder. Neuropsychiatr Dis Treat. 2006;2:427-43.

43. Muneer A. The treatment of adult bipolar disorder with aripiprazole: a systematic review. Cureus. 2016;8:e562.

44. Waage-Baudet H, Lauder JM, Dehart DB, Kluckman K, Hiller S, Tint GS, et al. Abnormal serotonergic development in a mouse model for the Smith-Lemli-Opitz syndrome: implications for autism. Int J Dev Neurosci. 2003;21:451-9.

45. Lee RW, Yoshida S, Jung ES, Mori S, Baker EH, Porter FD. Corpus callosum measurements correlate with developmental delay in Smith-Lemli-Opitz syndrome. Pediatr Neurol. 2013;49:107-12.

46. Boland MR, Tatonetti NP. Investigation of 7-dehydrocholesterol reductase pathway to elucidate off-target prenatal effects of pharmaceuticals: a systematic review. Pharm J. 2016;16:411-29. 